\title{
Small Angle Neutron Scattering Study of Fractal Structure of Oppositely Charged Nanoparticle and Protein Complexes
}

\author{
A. J. Chinchalikar ${ }^{1}$, V. K. Aswal ${ }^{1, *}$, J. Kohlbrecher ${ }^{2}$, and A. G. Wagh ${ }^{1}$ \\ ${ }^{1}$ Solid State Physics Division, Bhabha Atomic Research Centre, Mumbai 400 085, India \\ ${ }^{2}$ Laboratory for Neutron Scattering, Paul Scherrer Institut, CH5232 PSI Villigen, Switzerland
}

The structure of complexes of oppositely charged anionic silica nanoparticles and cationic lysozyme protein in aqueous solutions has been studied by small-angle neutron scattering. The measurements have been carried out for three different sized silica nanoparticles $(9.4,17.4$ and $28 \mathrm{~nm}$ ) at their fixed concentration (1 wt\%) while varying the concentration of lysozyme protein in the range $0-5 \mathrm{wt} \%$. The protein adsorption on the nanoparticles is governed by the attractive electrostatic interaction between nanoparticle and protein, which in turn lead to the aggregation of nanoparticles mediated by proteins. These aggregates are characterized by the fractal structure. The amount of adsorption and the evolution of fractal structure have been found to be strongly depending on the protein concentration and nanoparticle size.

KEYWORDS: Nanoparticles, Nanoparticle-Protein Interaction, Fractal Structure, SANS.

\section{INTRODUCTION}

The nanomaterials, due to their unique properties, are revolutionizing many areas in science and technology. ${ }^{1,2}$ These materials give rise to significantly different optical, electronic, magnetic and chemical properties from their bulk. ${ }^{3-6}$ There are different forms of nanomaterials (e.g., nanoparticles, nanotubes, nanorods etc.) out of which the nanoparticles are most commonly used having all the three dimensions in nano regime., ${ }^{7} 8$ The applications of nanoparticles cover broad range of areas including renewable energy, textiles, food, pharmaceuticals etc. ${ }^{9-12}$ Many of these applications involve the binding of various macromolecules like micelles, polymer or proteins to the nanoparticle. ${ }^{13-16}$ The surfactant-nanoparticle binding for example is utilized in detergency, colloidal stability and designing nanostructured functional materials. ${ }^{14,17}$ Another interest comes from the applications in targeted drug delivery, biosensing, diagnostics in which protein molecules bind with nanoparticle surface (known as protein corona) to reach to the affected location in the cell. ${ }^{18,19}$ The surface properties of nanoparticles can be tuned by attaching the different polymers to the nanoparticle surface. ${ }^{20,21}$ Thus, the nature

\footnotetext{
*Author to whom correspondence should be addressed

Email: vkaswal@barc.gov.in

Received: 26 February 2013

Accepted: 18 March 2013
}

of macromolecule-nanoparticle binding strongly depends upon the individual properties of components.

The interaction among different components involves forces like van der Waals, electrostatic, hydrophobic, covalent, hydrogen bonding etc. The change in each of these can give rise to variety of structures. The proteins can interact by different ways with the nanoparticles due to their labile nature and their ability to acquire various conformations. Many experimental techniques are being employed for characterization of protein corona. The circular dichroism (CD) spectroscopy has been used to determine the change in secondary structure of proteins on adsorption ${ }^{24,25}$ while differential scanning calorimetry (DSC) is used for detecting the phase changes at native temperatures. ${ }^{26,27}$ The dynamic light scattering (DLS) along with Fourier Transform Infrared Spectroscopy (FTIR) have been used to show various conformations acquired by the BSA protein on gold nanoparticles over the entire $\mathrm{pH}$ range. ${ }^{28}$ Sometimes, the adsorption is unwanted for certain medical devices which can be achieved by for example the PEGylation of the nanoparticle surface. ${ }^{29}$ The aim of present work is to study charge driven protein adsorption on nanoparticles and to study the overall structures of their complexes by smallangle neutron scattering (SANS). Although these proteinnanoparticle studies are in vitro, the future directions will require studies in vivo where the binding is much more enhanced and requires accounting more complex solution environments. 
SANS is a powerful technique to probe structure as well as interaction in colloidal systems. The ability to vary the contrast makes this technique unique in particular in order to study multi-component systems. ${ }^{30}$ We have studied the complexes of different sized Ludox silica nanoparticles with the lysozyme protein at physiological $\mathrm{pH}$ where two components are oppositely charged. Lysozyme is a small globular protein (M.W. $14.6 \mathrm{kDa}$ ) and most commonly studied model protein in the literature. The strong electrostatic interaction between oppositely charged nanoparticle and protein leads to the aggregation of nanoparticles and this phenomenon has been systematically characterized by SANS.

\section{EXPERIMENTAL SECTION}

The Ludox silica nanoparticles of different sizes (SM30, HS40 and TM40) and the Fluka hen egg white lysozyme (three times crystallized and lyophilized) were used as obtained. The Ludox silica nanoparticles are electrostatically stabilized where SM30, HS40 and TM40 systems correspond to 30,40 and $40 \mathrm{wt} \%$ concentrations of particles in water having particle surface area of approximately 360,220 and $140 \mathrm{~m}^{2} / \mathrm{g}$, respectively. The samples for SANS measurements were prepared by dissolving known amounts of nanoparticle and protein in $20 \mathrm{mM}$ phosphate buffer $(\mathrm{pH} 7)$ solution. Both $\mathrm{D}_{2} \mathrm{O}$ and $\mathrm{H}_{2} \mathrm{O}$ were used as a solvent to obtain different scattering contrast of the components in SANS experiments. Smallangle neutron scattering measurements were carried out at the SANS-I facility, Swiss Spallation Neutron Source SINQ, Paul Scherrer Institut, Switzerland. ${ }^{31}$ The wavelength of neutron beam used was $8 \AA$. The experiments were performed at two sample-to-detector distances 2 and $8 \mathrm{~m}$ to cover wave vector transfer $Q(=4 \pi \sin \theta / \lambda$, where $2 \theta$ is the scattering angle and $\lambda$ is the wavelength of incident neutron) in the range of 0.005 to $0.25 \AA^{-1}$. The scattered neutrons were detected using two-dimensional $96 \mathrm{~cm}$ $\times 96 \mathrm{~cm}$ detector. All the measurements were performed at the fixed concentration (1 wt\%) of silica nanoparticles with the lysozme concentrations varied over the range of 0 to $5 \mathrm{wt} \%$. Samples were held in $1 \mathrm{~mm}$ thick Hellma quartz cells during the experiments. All the data were corrected and normalized to absolute scale using BERSANS-PC data processing software. ${ }^{32}$

\section{SMALL-ANGLE NEUTRON SCATTERING ANALYSIS}

SANS is an elastic scattering technique in which one measures coherent differential scattering cross section per unit volume $(d \Sigma / d \Omega)$ as a function of $Q$. For a monodispersed system of particles, $d \Sigma / d \Omega$ can be expressed as ${ }^{33,34}$

$$
\frac{d \Sigma}{d \Omega}(Q)=n\left(\rho_{p}-\rho_{s}\right)^{2} V_{p}^{2} P(Q) S(Q)+B
$$

where $n$ is the number density of particles, $\rho_{p}$ and $\rho_{s}$ are scattering length densities of particle and solvent, respectively. $P(Q)$ is intra-particle structure factor which contains information about shape and size of particle. $S(Q)$ represents inter-particle structure factor and decided by the interaction between particles. $B$ is a constant term due to incoherent background which is mainly from hydrogen content in the sample.

$P(Q)$ for the spherical particle of radius $R$ is given by

$$
P(Q)=\left[\frac{3\{\sin (Q R)-Q R \cos (Q R)\}}{(Q R)^{3}}\right]^{2}
$$

In the case of ellipsoidal particle, $P(Q)$ is calculated by

$$
\begin{gathered}
P(Q)=\int_{0}^{1} F^{2}(Q, \mu) d \mu \\
F(Q, \mu)=\frac{3(\sin x-x \cos x)}{x^{3}} \\
x=Q\left[a^{2} \mu^{2}+b^{2}\left(1-\mu^{2}\right)\right]^{1 / 2}
\end{gathered}
$$

where $a$ and $b=c$ are semi-major and semi-minor axes of particle. $V_{c}\left(=4 \pi a b^{2} / 3\right)$ is the total volume of the particle. The variable $\mu$ is the cosine of the angle between the directions of $a$ and $Q$.

The size polydispersity of particles is included in the expression of differential scattering cross section as ${ }^{35}$

$$
\frac{d \Sigma}{d \Omega}(Q)=\int \frac{d \Sigma}{d \Omega}(Q, R) f(R) d R+B
$$

where $f(R)$ is size distribution and is usually accounted by $\log$ normal distribution (median radius $=R_{m}$ and width $=w$ ) as given by

$$
f(R)=\frac{1}{R w \sqrt{2 \pi}} \exp \left[-\frac{\ln \left(R / R_{m}\right)}{2 w^{2}}\right]
$$

The scattering cross section for the particle aggregates as characterized by fractal structure is expressed by ${ }^{36}$

$$
\frac{d \Sigma}{d \Omega}(Q)=n_{a} V_{p}\left(\rho_{p}-\rho_{s}\right)^{2} P_{b}(Q) S_{f}(Q)+B
$$

where $n_{a}$ and $V_{p}$ are the number density and volume of individual scatterer in the aggregates. $P_{b}(Q)$ is the intraparticle structure factor of the building block in aggregated structure and $S_{f}(Q)$ is the structure factor. $S_{f}(Q)$ for mass fractal is given by the following ${ }^{36}$

$$
\begin{aligned}
S_{f}(Q)= & +\frac{1}{(Q R)^{D}} \frac{D \Gamma(D-1)}{\left[1+\left(1 /(Q \xi)^{2}\right)\right]^{(D-1) / 2}} \sin \{(D-1) \\
& \left.\times \tan ^{-1}(Q \xi)\right\}
\end{aligned}
$$

where $\xi$ signifies the maximum length up to which fractal microstructure exists and $D$ is the fractal dimension. 


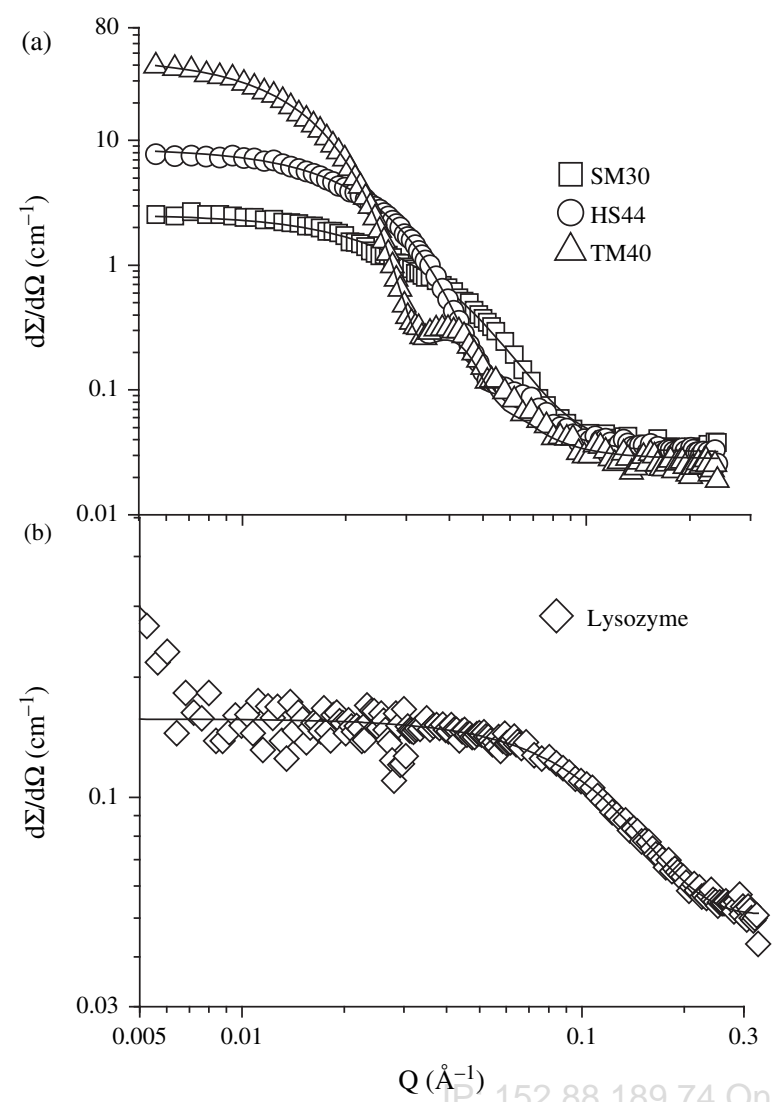

Fig. 1. SANS data of $1 \mathrm{wt} \%$ (a) silica nanoparticles (SM30, HS40 and TM40) and (b) lysozyme in aqueous $\left(\mathrm{D}_{2} \mathrm{O}\right)$ buffer solution at $\mathrm{pH} 7$.

The corrections were made during the data analysis for the instrumental smearing where calculated scattering profiles are smeared by the appropriate resolution function to compare with the measured data. The parameters in the analysis were optimized by means of nonlinear least-squares fitting program. ${ }^{37}$

\section{RESULTS AND DISCUSSION}

Figure 1 shows the SANS data of pure 1 wt $\%$ silica nanoparticles (SM30, HS40, TM40) and 1 wt\% lysozyme

Table I. The calculated parameters of 1 wt $\%$ silica nanoparticles (SM30, HS40 and TM40) and $1 \mathrm{wt} \%$ lysozyme protein systems.

\begin{tabular}{lcc}
\hline \multicolumn{3}{c}{ (a) Silica nanoparticles } \\
\hline Particle & Mean size $(\mathrm{nm})$ & Polydispersity \\
\hline SM30 & 9.4 & 0.34 \\
HS40 & 17.4 & 0.21 \\
TM40 & 28.0 & 0.14 \\
\hline
\end{tabular}

(b) Lysozyme protein

\begin{tabular}{lcc}
\hline Particle & Semi-major axis $(\mathrm{nm})$ & Semi-minor axis $(\mathrm{nm})$ \\
\hline Lysozyme & 2.4 & 1.25 \\
\hline
\end{tabular}

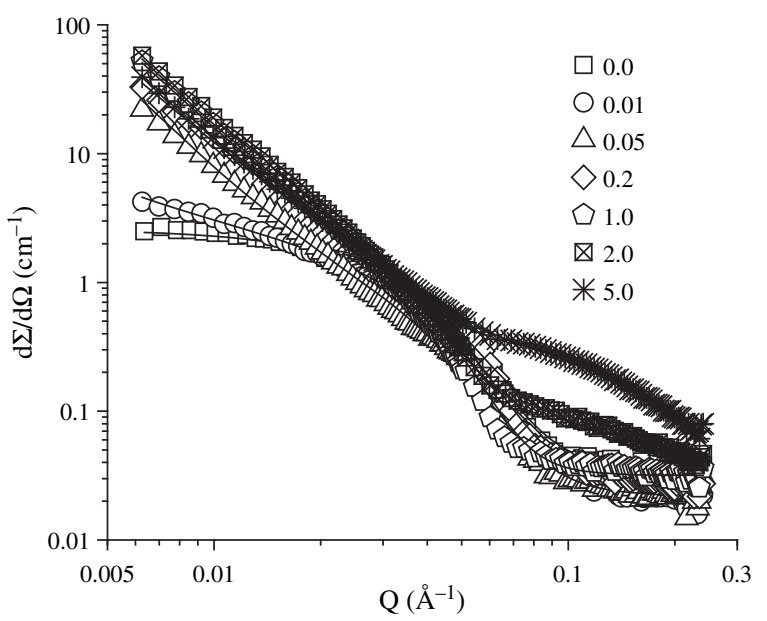

Fig. 2. SANS data of $1 \mathrm{wt} \% \mathrm{SM} 30$ silica nanoparticle system at different lysozyme concentrations in $\mathrm{D}_{2} \mathrm{O}$ buffer at $\mathrm{pH} 7$.

in aqueous $\left(\mathrm{D}_{2} \mathrm{O}\right)$ solution at $\mathrm{pH}$ 7. All these data do not show any direct signature of structure factor. Further, it was observed that the data can be scaled with those of lower concentrations, confirming the scattering in Figure 1 mostly arise from the form factor contributions. The data of silica nanoparticles show different magnitude and width of the scattering profiles indicate the significantly different sizes of SM30, HS40 and TM40 nanoparticles. The humps in the SANS data can arise due to the form factor oscillations of the particle whose visibility depends on their smearing by combined effect of polydispsersity, instrument resolution and scattering background. ${ }^{38}$ All the data have been fitted by considering systems dilute $(\mathrm{S}(\mathrm{Q}) \sim 1)$. The silica nanoparticle data were modeled for polydispersed spheres using Eq. (6) and fitted parameters are given in Table I. The fitted values of nanoparticle sizes (diameter) are 9.4, 17.4 and $28 \mathrm{~nm}$ having corresponding polydispersities 0.34, 0.21 and 0.14 for SM30, HS40 and TM40, respectively. The lysozyme is a small globular protein and has been modeled by prolate ellipsoidal shape with dimensions $4.8 \times 2.5 \times 2.5 \mathrm{~nm}^{3} .{ }^{39,40}$ The deviation in the data of lysozyme protein from the fitting line in low $\mathrm{Q}$ region is believed to be originating from permanent aggregates usually found in protein solutions. ${ }^{41}$

The SANS data of $1 \mathrm{wt} \%$ SM30 silica nanoparticles in $\mathrm{D}_{2} \mathrm{O}$ at $\mathrm{pH} 7$ with varying concentration of lysozyme are shown in Figure 2. These data show the scattering buildup in the low as well as high $Q$ regions with the increase in the lysozyme concentration. The scattering build-up in low $Q$ region can be expected for system undergoing structural and/or interactional changes. ${ }^{42}$ The fact that the measurements have been done with low concentration of nanoparticles makes it difficult to probe the interactional changes. Moreover, it has been observed that the system becomes turbid in presence of protein suggesting the formation of large aggregates in these systems. Most of the data (beyond 0.02 wt\% lysozyme concentration) 


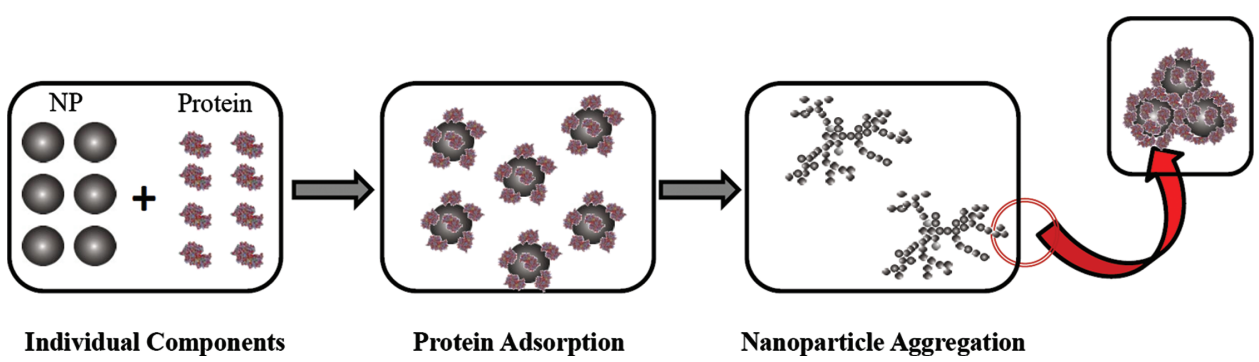

Fig. 3. A schematic of aggregation of silica nanoparticles in presence of lysozyme protein.

Table II. The fitting parameters of SANS data of $1 \mathrm{wt} \%$ SM30 silica nanoparticle system with varying concentration of lysozyme. The fitted value of fractal dimension irrespective of protein concentration is 2.4.

\begin{tabular}{lccccccc}
\hline \multicolumn{7}{c}{ (i) Low protein concentration regime } \\
\hline Protein concentration (wt \%) & 0.01 & 0.02 & 0.05 & 0.1 & 0.2 & 0.5 \\
Free silica fraction (\%) & 93 & 86 & 70 & 35 & 20 & 0 \\
\hline
\end{tabular}

(ii) High protein concentration regime

Protein concentration (wt\%) $\quad \begin{array}{lll}1.0 & 2.0 & 5.0\end{array}$

Free protein fraction $(\%) \quad 30 \quad 60 \quad 84$

show linearity over a wide Q range (more than one order of magnitude of $\mathrm{Q}$ ) on $\log -\log$ scale which support to the fractal aggregation of nanoparticles. ${ }^{43,44}$. The aggregation of silica nanoparticles is believed to be mediated by the adsorption of oppositely charged lysozyme proteins on nanoparticles through strong attractive electrostatic interaction between them. A schematic of proposed aggregation mechanism is shown in Figure 3. The build-up of scattering in the high $Q$ region, in particular, at high protein concentrations is as a result of excess proteins (not used in the nanoparticle aggregation) coexisting with the nanoparticle aggregates. The data are fitted combining Eqs. (1) and (8), whose fitted parameters are given in Table II. The data

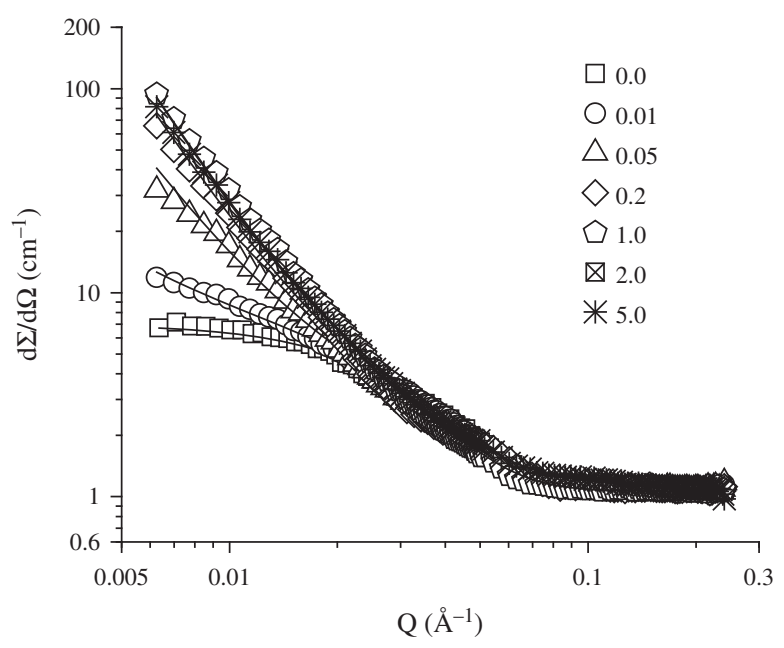

Fig. 4. SANS data of $1 \mathrm{wt} \%$ SM30 silica nanoparticle system with different lysozyme concentrations in $\mathrm{H}_{2} \mathrm{O}$ buffer at $\mathrm{pH} 7$. fitting for low protein concentrations is carried out by considering two populations consisting of aggregates of silica nanoparticles mediated by oppositely charged lysozyme protein and free silica nanoparticles. The aggregates are characterized by the mass fractal having fractal dimension of about 2.4. Irrespective of protein concentration, the constant value of fractal dimension (2.4) indicates diffusion limited aggregation (DLA) type of fractal morphology of the aggregates. ${ }^{45,46}$ The calculated percentage of free silica nanoparticles decreases with increase in protein concentration. The value of minimum protein concentration required for the aggregation of all the silica nanoparticles $(1 \mathrm{wt} \%)$ is found to be $0.5 \mathrm{wt} \%$. At higher protein

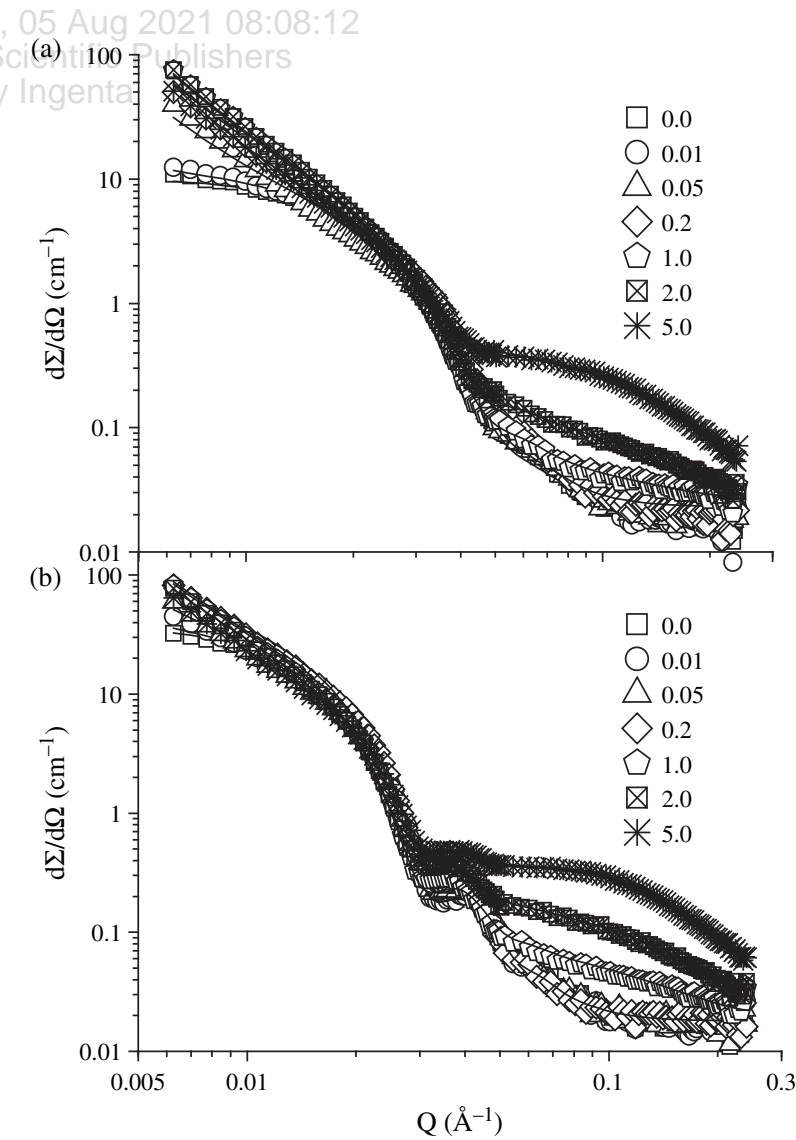

Fig. 5. SANS data of $1 \mathrm{wt} \%$ (a) HS40 and (b) TM40 silica nanoparticle systems with different lysozyme concentrations in $\mathrm{D}_{2} \mathrm{O}$ buffer at $\mathrm{pH} 7$. 
Table III. The fitting parameters of SANS data of $1 \mathrm{wt} \%$ (a) HS40 and (b) TM40 silica nanoparticle system with varying concentration of lysozyme. The fitted value of fractal dimension is 2.4 .

(a) HS40 silica nanoparticle system

\begin{tabular}{lccccc}
\hline & (i) Low protein concentration regime & 0.05 & 0.1 & 0.2 \\
Protein concentration (wt $\%)$ & 0.01 & 0.02 & 40 & 30 & 10 \\
Free silica fraction (\%) & 87 & 73 & (ii) High protein concentration regime & 0.5 \\
& & 2.0 & 5.0 & 86 \\
Protein concentration (wt $\%)$ & 1.0 & 65 & 34 & &
\end{tabular}

Free protein fraction $(\%)$

(b) TM40 silica nanoparticle system

Protein concentration (wt \%)

Free silica fraction (\%)

Protein concentration (wt $\%$ )

Free protein fraction $(\%)$ (i) Low protein concentration regime

$0.02 \quad 0.05$

60

0.05
30

0.1

10

0.2

0

0.5

(ii) High protein concentration regime

1.0

38
2.0

68
5.0

87 concentrations ( $>0.5 \mathrm{wt} \%)$, data are analyzed with aggregates of nanoparticles coexisting with excess free proteins. The free protein concentration increases with increasing protein concentration.

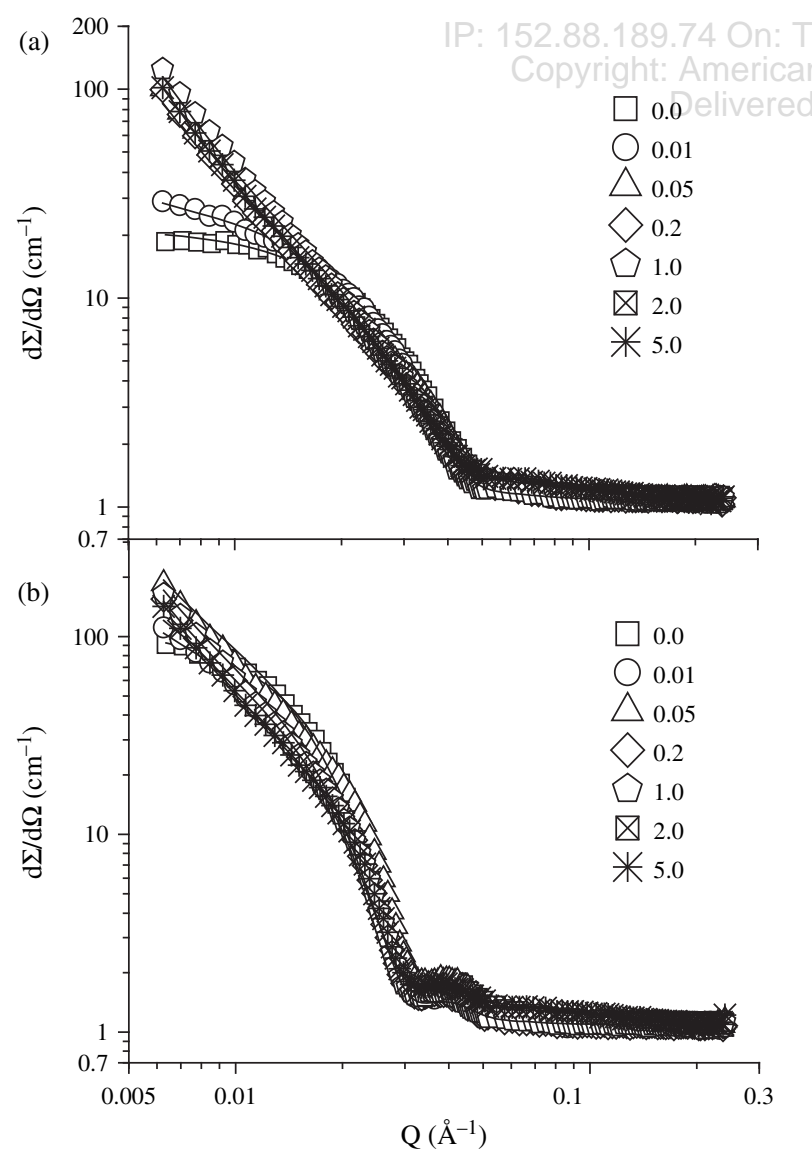

Fig. 6. SANS data of $1 \mathrm{wt} \%$ (a) HS40 and (b) TM40 silica nanoparticle system with varying lysozyme concentrations in $\mathrm{H}_{2} \mathrm{O}$ buffer at $\mathrm{pH}$.
The SANS data of the system corresponding to Figure 2 for changing the buffer from $\mathrm{D}_{2} \mathrm{O}$ to $\mathrm{H}_{2} \mathrm{O}$ are shown in Figure 4. The use of $\mathrm{H}_{2} \mathrm{O}$ enhances contrast to silica nanoparticles whereas suppresses the scattering from protein. Therefore, the scattering in this system mostly arise from the nanoparticles. As a result, the scattering show a build-up only in the low $\mathrm{Q}$ region, related to the aggregation of nanoparticles. The absence of scattering build-up at higher $Q$ region (unlike Fig. 2) confirms the coexistence of free proteins with nanoparticle aggregates. The data in Figure $4\left(\mathrm{H}_{2} \mathrm{O}\right.$ as solvent) are fitted with the same parameters of fractal structure of nanoparticles aggregates $\left(\mathrm{D}_{2} \mathrm{O}\right.$ as solvent) and found to be in good agreement. It is found that irrespective of the protein concentration the structure of nanoparticle aggregates is same. The protein concentration decides the fraction of nanoparticles undergoing aggregation as well as amount of free protein if any.

The size dependent interaction of nanoparticle with protein has been studied in Figure 5. The data are shown for two different sized $1 \mathrm{wt} \%$ silica nanoparticles (HS40 and TM40) with varying concentration of lysozyme in $\mathrm{D}_{2} \mathrm{O}$ buffer solution ( $\mathrm{pH} 7)$. The data for both the nanoparticle show similar protein concentration dependent scattering features as has been observed with SM30 nanoparticles (Fig. 2). However, the evolution of structure in these systems depends on the size of nanoparticle. For example, the build-up of scattering in the low $Q$ region (related to particle aggregation) is enhanced with the increase in the size of the particle. The particle aggregation for larger sized nanoparticles can be seen at much lower protein concentrations. The fitted structural parameters are given in Table III. The fraction of aggregated nanoparticles increases with the protein concentration and is found to be prominent for the larger sized nanoparticles. It is also found that the fraction of free proteins at higher protein concentrations increases with the increase in the 
Table IV. The comparison of structure of SM30, HS40 and TM40 silica nanoparticle systems at three different regions of protein concentrations.

\begin{tabular}{|c|c|c|c|c|c|}
\hline & & & $1 \mathrm{wt} \% \mathrm{SM} 30 / \mathrm{HS} 40 / \mathrm{TM} 40+$ & x wt\% lysozyme & \\
\hline \multirow[b]{3}{*}{$\begin{array}{l}\text { Nanoparticle } \\
\text { system }\end{array}$} & \multirow[b]{3}{*}{$\begin{array}{l}\text { Nanoparticle } \\
\text { size }(\mathrm{nm})\end{array}$} & \multirow[b]{3}{*}{$\begin{array}{l}\text { Fractal dimension } \\
\text { of aggregates }\end{array}$} & \multicolumn{3}{|c|}{$\begin{array}{c}\text { Lysozyme } \\
\text { concentration (wt } \%)\end{array}$} \\
\hline & & & 0.05 & 0.5 & 5.0 \\
\hline & & & $\begin{array}{c}\text { Free silica } \\
\text { fraction }(\%)\end{array}$ & $\begin{array}{c}\text { Free silica/ } \\
\text { protein fraction }(\%)\end{array}$ & $\begin{array}{l}\text { Free protein } \\
\text { fraction }(\%)\end{array}$ \\
\hline SM30 & 9.4 & 2.4 & 70 & 0 & 84 \\
\hline HS40 & 17.4 & 2.4 & 40 & 0 & 86 \\
\hline TM40 & 28 & 2.4 & 30 & 0 & 87 \\
\hline System structure & & & Free silica + Fractal aggregates & Fractal aggregates & Fractal aggregates + Free proteins \\
\hline
\end{tabular}

size of the particle, which is expected as the total surface area of particles at given volume fraction decreases. The fractal dimension of the particle aggregates irrespective of the size is found to be same about 2.4. All these aggregation are governed by the DLA, as a result lead to the same fractal structure irrespective of the size of the nanoparticles.

The SANS data for $1 \mathrm{wt} \%$ HS40 and TM40 nanoparticle systems in $\mathrm{H}_{2} \mathrm{O}(\mathrm{pH} 7)$ are shown in Figure 6. In $\mathrm{H}_{2} \mathrm{O}$ as solvent, the scattering from proteins is minimized and hence the corresponding changes in data arise exclusively as a result of change in the structure of nanoparticle assembly. The observed features are consistent with SANS data of $\mathrm{D}_{2} \mathrm{O}$ as solvent, where the particle aggregation mediated by protein depends on the size of the nanoparticle. The data fitting is carried out by neglecting the scattering from proteins and using the same parameters as in Table III. The fraction of aggregated nanoparticles increases with the protein concentration with prominent effect for the larger sized nanoparticles. The fractal dimension of the particle aggregates has a value about 2.4 for all the nanoparticles.

The protein concentration dependent comparison of interaction of nanoparticle with protein for different sized nanoparticles is given in Table IV. The interaction of two components is compared for three different regions of protein concentrations where

(i) nanoparticle aggregates coexist with free nanoparticles at low protein concentrations,

(ii) all the nanoparticle have been aggregated for the intermediate concentration range of protein and

(iii) excess protein coexists with nanoparticle aggregates at higher protein concentrations.

In the first region of low protein concentration (e.g., $0.05 \mathrm{wt} \%$ ), the fact that the total surface area of the particles (available for adsorption) decreases with increasing size suggests that amount of protein required for neutralizing the charge on the nanoparticles will be less and hence easier to aggregate them. This gives rise to decrease in the fraction of free nanoparticles (coexisting with aggregates) at low protein concentration with increase in the particle size. In the second region of protein concentration (e.g., $0.5 \mathrm{wt} \%$ ) where all the nanoparticles have been aggregated, the scattering data is governed by the fractal structure of nanoparticle aggregates. The diffusion limited aggregation results in similar aggregated structures (fractal dimension $~ 2.4$ ) irrespective of the nanoparticle size in these systems. A constant value of fractal dimension is found because mechanism of aggregation (chargedriven) and particle geometry (spherical) are same in these nanoparticle systems. The similar results can be examined by the computer simulations. ${ }^{47-49}$ The free protein coexist with nanoparticle aggregates at higher protein concentrations (e.g., 5 wt $\%$ ) in the third region. The increase in the amount of free protein with increase in nanoparticle size is expected due to lesser available surface area for the adsorption. These studies thus suggest that nanoparticleprotein interaction can be tuned by the varying the size of the nanoparticle.

\section{CONCLUSIONS}

Small-angle neutron scattering study has been performed to examine the interaction and resultant structure of anionic silica nanoparticles with oppositely charged lysozyme protein in aqueous solution. The protein adsorption on nanoparticle leads to aggregation of nanoparticles. The amount of protein adsorption can be tuned by varying the protein concentration as well as by the size of the nanoparticle. The nanoparticle aggregates coexist with the individual nanoparticles at low protein concentrations whereas free protein and nanoparticle aggregates coexist at higher protein concentrations. The amount of protein adsorption per nanoparticle increases dramatically with the increase in the nanoparticle size. On the other hand, the overall adsorption decreases with the increase in the size of the nanoparticle. The aggregates are characterized by the mass fractal governed by diffusion limited aggregation.

Acknowledgments: This work is based on the SANS experiments performed at the Swiss Spallation Neutron Source SINQ, Paul Scherrer Institut, Villigen, Switzerland. 


\section{References and Notes}

1. V. L. Colvin, Nat. Biotechnol. 21, 1166 (2003).

2. I. Lynch, T. Cedervall, M. Lundqvist, C. Cabaleiro-Lago, S. Linse, and K. A. Dawson, Adv. Colloid. Interface Sci. 134, 167 (2007).

3. C. J. Murphy, A. M. Gole, J. W. Stone, P. N. Sisco, A. M. Alkilany, E. C. Goldsmith, and S. C. Baxter, Acc. Chem. Res. 41, 1721 (2008).

4. H. Gleiter, J. Weissmuller, O. Wollersheim, and R. Wurschum, Acta Materialia 49, 737 (2001).

5. D. L. Leslie-Pelecky and R. D. Rieke, Chem. Mater. 8, 1770 (1996).

6. L. Lizana, Z. Konkoli, B. Bauer, A. Jesorka, and O. Orwar, Annu. Rev. Phys. Chem. 60, 449 (2009)

7. M. Malmsten, Biopolymers at Interfaces, Dekker, New York (2003).

8. C. M. Niemeter, Angew. Chem., Intl. 40, 4128 (2001).

9. D. J. Maxwell, J. R. Taylor, and S. Nie, J. Am. Chem. Soc. 124, 9606 (2002).

10. C. C. You, A. Verma, and V. M. Rotello, Soft Matter 2, 190 (2006).

11. N. Sozer and J. L. Kokini, Trends in Biotechnology 27, 82 (2009).

12. S. S. Mao and X. Chen, Internat. J. Energy Res. 31, 619 (2007).

13. M. J. Rosen, Surfactants in emerging technologies, Dekker, New York (1987).

14. R. Zhang and P. Somasundaran, Adv. Collod. Interface Sci. 123, 213 (2006).

15. F. Zhang, E. Lees, G. P. Rivera, F. Yang, P. Mulvaney, and W. J. Parak, Small 7, 3113 (2011).

16. J. Klein, Proc. Nat. Acad. Sci. 107, 2029 (2007).

17. S. Kumar and V. K. Aswal, J. Phys.: Condens. Matt. 23, 035101 (2011).

18. D. K. Malik, S. Baboota, A. Ahuja, S. Hasan, and J. Ali, Curr. drug delivery 4, 141 (2007).

19. I. Lynch and K. A. Dawson, Nanotoday 3, 40 (2008).

20. B. J. Kim, G. H. Fredrickson, J. Bang, C. J. Hawker, and E. J. Kramer, Macromolecules 42, 6193 (2009).

21. Y. Wang, J. Zhang, F. Zhu, R. Zhou, C. Shi, J. Ping, Z. Cheng, and H. Ni, J. Poly. Mat. 22, 267 (2005).

22. J. N. Israelachvili, Intermolecular and Surface Forces, Academic Press, New York (1992).

23. C. N. Likos, Phys. reports 348, 267 (2001).

24. S. M. Kelly and N. C. Price, Curr. Protein and Peptide Sci. 1, 349 (2000)
25. M. E. Aubin-Tam and K. H. Schifferli, Langmuir 21, 12080 (2005).

26. E. Casals, T. Pfaller, A. Duschl, G. J. Oostingh, and V. Puntes, ACS Nano 4, 3623 (2010).

27. J. H. Teichroeb, J. A. Forest, V. Ngai, and L. W. Jones, Euro. Phys. J. E 21, 19 (2006).

28. D. H. Tsai, F. W. DelRio, A. M. Keene, K. M. Tyner, R. I. MacCuspie, T. J. Cho, M. R. Zachariah, and V. A. Hackley, Langmuir 27, 2464 (2011).

29. W. Norde and D. Gage, Langmuir 20, 4162 (2004).

30. S. H. Chen, Annu. Rev. Phys. Chem. 37,351 (1986).

31. J. Kohlbrecher and W. Wagner, J. Appl. Cryst. 33, 804 (2000)

32. U. Keiderling, Appl. Phys. A: Mater. Sci. Process. 74, s1455 (2002).

33. J. S. Pedersen and C. Svaneborg, Curr. Opinion Colloid Interface Sci. 7, 158 (2002).

34. J. B. Hayter and J. Penfold, Colloid Polymer Sci. 261, 1022 (1983).

35. V. K. Aswal, P. S. Goyal, and P. Thiyagarajan, J. Phys. Chem. 102, 2469 (1998)

36. J. Teixeira, J. Appl. Cryst. 21, 781 (1988).

37. J. S. Pederson, Adv. Colloid. Interface Sci. 70, 171 (1997).

38. M. Kotlarchyk and S. H. Chen, J. Chem. Phys. 79, 2461 (1983).

39. Y. Liu, E. Fratini, P. Baglioni, W. R. Chen, and S. H. Chen, Phys. Rev. Lett. 95, 118102 (2005).

40. A. J. Chinchalikar, S. Kumar, V. K. Aswal, P. Callow, and A. G. Wagh, Am. Inst. Phys. Conf. Proc. 1447, 161 (2012).

41. A. Shukla, E. Mylonas, E. Di Cola, S. Finet, P. Timmins, T. Narayanan, and D. I. Svergun, Proc. Nat. Acad. Sci. 105, 5075 (2008).

42. T. Freltoft, J. K. Kjems, and S. K. Sinha, Phys. Rev. B 33, 269 (1986).

43. D. F. R. Mildner and P. L. Hall, J. Phys. D: Appl. Phys. 19, 1535 (1986).

44. S. Kumar, V. K. Aswal, and J. Kohlbrecher, Langmuir 27, 10167 (2011).

45. T. A. Witten and L. M. Sander, Phys. Rev. Lett. 47, 1400 (1981).

46. X. Z. Wang and Y. Huang, Phys. Rev. A 46, 5038 (1992).

47. P. Meakin, Adv. Colloid Interface Sci. 28, 249 (1988).

48. D. Li, W. Y. Ting, and O. Y. Zhong-Can, Commun. Theor. Phys. 58, 895 (2012).

49. A. Menshutin, Phys. Rev. Lett. 108, 15501 (2012). 